\title{
Genetic predisposition for sudden cardiac death in myocardial ischaemia: the Arrhythmia Genetics in the NEtherlandS study
}

\author{
R. F. J. Marsman • A. A. M. Wilde • C. R. Bezzina
}

Published online: 28 January 2011

(C) The Author(s) 2011. This article is published with open access at Springerlink.com

\begin{abstract}
Sudden cardiac death from ventricular fibrillation during myocardial infarction is a leading cause of total and cardiovascular mortality. This multifactorial, complex condition clusters in families, suggesting a substantial genetic cause. We carried out a genomewide association study (GWAS) for sudden cardiac death, in the AGNES (Arrhythmia Genetics in the Netherlands) population, consisting of patients with (cases) and without (controls) ventricular fibrillation during a first ST-elevation myocardial infarction. The most significant association was found at chromosome $21 \mathrm{q} 21$ (rs2824292; odds ratio $=1.78$, 95\% CI $1.47-$ 2.13, $\left.P=3.3 \times 10^{-10}\right), 98 \mathrm{~kb}$ proximal of the $C X A D R$ gene, encoding the Coxsackie and adenovirus receptor. This locus has not previously been implicated in arrhythmia susceptibility. Further research on the mechanism of this locus will ultimately provide novel insight into arrhythmia mechanisms in this condition.
\end{abstract}

Keywords Sudden cardiac death · Ventricular fibrillation . Genomewide association study . Single nucleotide polymorphism

R. F. J. Marsman • A. A. M. Wilde • C. R. Bezzina ( $\triangle)$

Heart Failure Research Center, Department of Experimental

Cardiology, Academic Medical Center,

Meibergdreef 15,

1105 AZ, Amsterdam, the Netherlands

e-mail: c.r.bezzina@amc.uva.nl

A. A. M. Wilde

Department of Cardiology, Academic Medical Center,

Amsterdam, the Netherlands

\section{Introduction}

Sudden cardiac death (SCD) accounts for almost $20 \%$ of total mortality in Western Europe and the USA, and ventricular fibrillation (VF) is the most common underlying arrhythmia [1, 2]. VF arises through multiple mechanisms, depending on the underlying cardiac pathology. In the last decade, considerable progress has been made in the understanding of the genetic, molecular and electrophysiological basis of SCD in the uncommon (monogenic) familial arrhythmia syndromes affecting the structurally normal heart and the rare inherited structural disorders associated with increased SCD risk [3, 4]. However, the overwhelming majority $(\sim 80 \%)$ of SCDs in adults are caused by the sequelae of coronary artery disease, namely myocardial ischaemia or acute myocardial infarction (MI) [2], where SCD is the first clinically identified expression of heart disease in up to half of cases [5]. In contrast to the monogenic rhythm disorders, progress in understanding the molecular determinants of VF in these conditions, affecting a much greater proportion of the population, has been very limited [6]. Consequently, specific and sensitive risk predictors, as well as therapeutic targets for prevention, are currently missing. Because of the magnitude of the problem, the opportunities for a real public health impact of improved risk stratification and novel preventive therapeutic strategies are enormous. Even minor improvements could reduce the number of victims significantly.

\section{Heritability of SCD}

The concept of a heritable component in the determination of risk for SCD was first put forward by two epidemiological studies published in the late 1990s. In a population-based case- 
control study in the Seattle area, the rate of MI or SCD among first-degree relatives of cardiac arrest patients was almost $50 \%$ higher than that in first-degree relatives of control patients [7]. In the Paris Prospective Study, parental sudden death increased the relative risk of SCD among French men to 1.8, and sudden death of both parents increased the relative risk of SCD to 9.4 [8]. We subsequently analysed further the concept of genetic predisposition to SCD in a case-control study in which we selectively included only patients with a first, acute STelevation myocardial infarction (STEMI). In this population, recruited at multiple Dutch heart centres, which we named the Arrhythmia Genetics in the NEtherlandS - AGNES - study, we demonstrated that familial sudden death occurred significantly more frequently among STEMI patients who suffered VF (cases) compared with STEMI patients who did not suffer VF (controls) (OR 2.72, 95\% CI 1.84 to 4.03) [9]. This finding provided a strong rationale for the search of genetic modulators of risk in this specific cardiac pathology.

\section{Nature of Genetic Variation Modulating Risk}

VF during acute myocardial ischaemia can be regarded as a complex, multifactorial phenotype. There are two prevailing hypotheses as to the nature of the genetic variation modulating such common phenotypes [10]. The 'common disease-common variant hypothesis' implicates common genetic variants, present at frequencies of $>5 \%$ in the general population, such as single nucleotide polymorphisms (SNPs), each conferring a small to modest risk. The other hypothesis, the 'rare variant hypothesis', proposes that susceptibility to common disease is due to low frequency $(<5 \%)$ variants, each conferring a moderate increase in risk. The advent of arrays in the second half of the last decade, capable of genotyping hundreds of thousands of SNPs in an individual in one single experiment, enabled the assessment of the contribution of common genetic variation spread throughout the genome to complex diseases. A clear advantage of these genomewide association studies (GWAS) is that they are hypothesis-free, as there is no bias or list of candidate genes that are being tested. Genes previously unsuspected of being involved in the disease can therefore also be identified.

\section{A Genomewide Association Study of Ischaemia-Induced VF}

In order to identify common genetic variation underlying susceptibility to VF during a first acute MI, we recently conducted a genomewide association study in the AGNES case-control population [11]. The selection of one highly specific arrhythmia phenotype (mechanism) - for instance we excluded individuals with VF in the context of extensive pre-existing myocardial scarring from previous MIs - is critical in ensuring statistical power in such association studies. We genotyped 515 AGNES cases with $\mathrm{VF}$ and 457 AGNES controls without VF using the Illumina Human610-Quad array. After quality control of the genotype data, 507,436 SNPs were available for analysis. Non-genotyped SNPs throughout the genome were imputed by virtue of their degree of correlation to genotyped SNPs using data from the HapMap European CEU reference panel. The association of each SNP with VF was then evaluated using logistic regression assuming an additive genetic model with adjustment for age and sex. Over 2 million SNPs were evaluated in this way.

The distribution of $P$ values for the association of each SNP with VF is shown in Fig. 1. Eight SNPs clustering on chromosome 21q21 (the long arm of chromosome 21) showed the strongest association signal, passing the Bonferroni-corrected $P$ value threshold of $P<5 \times 10^{-8}$. From these, the most significant association signals were found for $\mathrm{rs} 2824292$ and $\operatorname{rs} 2824293\left(P=3.3 \times 10^{-10}\right)$. These two SNPs, which are highly correlated (in strong linkage disequilibrium) with each other, exhibited an odds ratio of 1.78 per copy of the $\mathrm{G}$ allele $(95 \%$ confidence interval, 1.47-2.13; risk allele was G for both SNPs). Approximately $30 \%$ of AGNES cases were homozygous for these alleles in contrast to only $15 \%$ of AGNES controls. As expected, the frequency of the risk alleles in the general population was intermediate between those of cases and controls (Fig. 1).

\section{Replication}

One of the SNPs (rs2824292) was then carried forward for replication in an independently recruited case-control set. Cases for replication $(n=150)$ were drawn from the AmsteRdam REsuscitation Study (ARREST-11). ARREST-11 is an ongoing prospective population-based study aimed at establishing the genetic, clinical and environmental determinants of SCD in the general population. Caucasian out-of-hospital cardiac arrest patients in the ARREST-11 dataset presenting with MI at hospital admission and with ECG-documented fast ventricular tachycardia or VF were included in the replication analysis. Controls for the replication analysis were MI survivors drawn from the GENetic DEterminants of Restenosis (GENDER) study [12] (GENDER-MI; $n=391$ ). The GENDER population comprises consecutive patients who underwent successful elective percutaneous transluminal coronary angioplasty at one of four Dutch heart centres. As observed in the AGNES case-control set, the risk G allele of SNP rs2824292 at chromosome $21 \mathrm{q} 21$ was more abundant and the protective A allele was less abundant in the ARREST-MI cases compared with the GENDER-MI controls. 
a

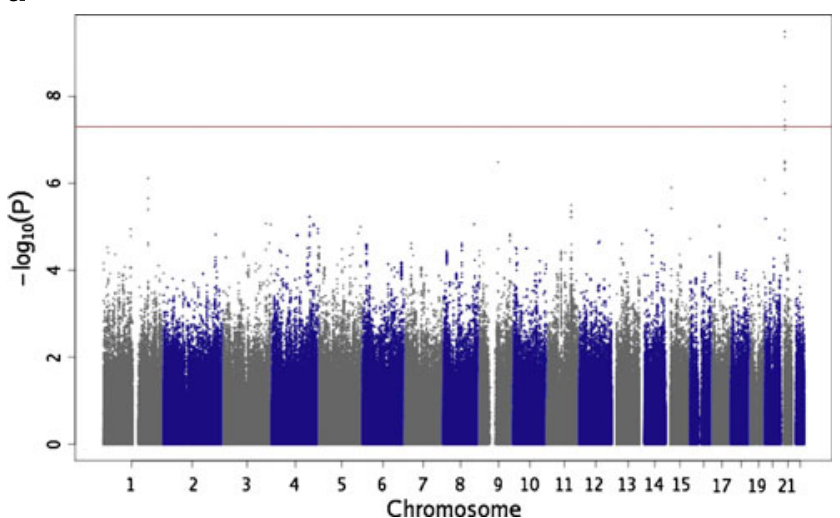

b

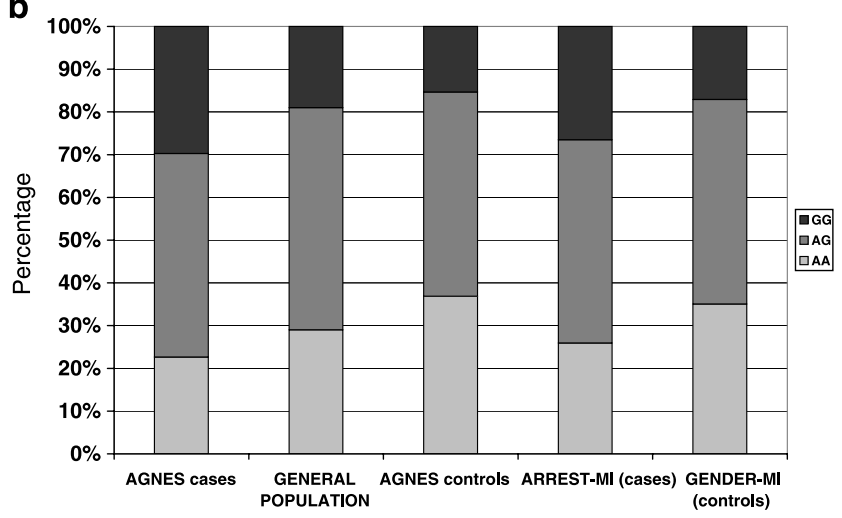

C

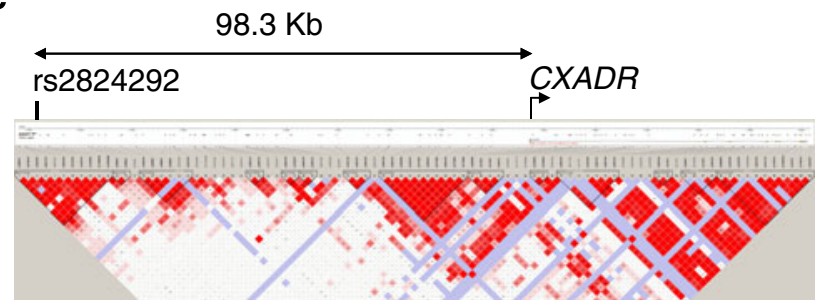

Fig. 1 Results of genomewide association analysis in the AGNES case-control population. a Manhattan plot of the genomewide association scan in the AGNES population. Each dot represents a different SNP, with the alternating bands of colour showing different chromosomes of the human genome ( $X$ axis). The negative logarithms of the $P$ values, expressing the strength of the association, are displayed on the $Y$ axis. $P$ values are corrected for age, sex and inflation factor. The horizontal red line indicates the preset threshold for genomewide significance, $P<5 \times 10^{-8}$. Data drawn from Bezzina et al. [11]. b Distribution of rs2824292 genotypes in the discovery set, the replication set and a sample of the general population. c Haploview plot of linkage disequilibrium $\left(\mathrm{D}^{\prime}\right)$ for all CEU HapMap SNPs in the region harbouring rs2824292 and the CXADR gene. Colour scheme: $\mathrm{D}^{\prime}=1$ and $\mathrm{LOD} \geq 2$ bright red, $\mathrm{D}^{\prime}<1$ and $\mathrm{LOD} \geq 2$ shades of pink/red, $\mathrm{D}^{\prime}=1$ and $\mathrm{LOD}<2$ blue, $\mathrm{D}^{\prime}<1$ and $\mathrm{LOD}<2$ white

\section{Chromosome 21q21 in Arrhythmia Susceptibility}

The chromosome 21q21 locus had not previously been linked to arrhythmia and the mechanism by which common genetic variation at this locus confers an increased risk to $\mathrm{VF}$ is unknown. Common genetic variation is expected to impact on disease either by changing the amino acid sequence of a protein (and thereby its function) or by impacting on the level of gene expression and consequently protein level. The SNPs at chromosome 21q21 impacting on risk for $\mathrm{VF}$ are not located within a gene. Inspection of a 1 megabase $(\mathrm{Mb})$ interval spanning both upstream and downstream of these SNPs identified three genes all located within a region of $0.5 \mathrm{Mb}$ downstream. One is BTG3, located at a distance of $179 \mathrm{~kb}$ from the association signal. This gene encodes B cell translocation gene 3, a member of the $P C 3, B T G$ and $T O B$ family of growth inhibitory genes [13]. Its mRNA expression level is relatively high in the testis, lung and ovary but is low in heart. Another gene in the region is C21orf91, located at $374 \mathrm{~kb}$ from the association signal; it encodes the human orthologue of the chicken C21orf91 (also known as EURL, encoding the early undifferentiated retina and lens protein), which is known to be expressed in the developing chick retina and lens and has been suggested to play a role in the development of these structures [14].

The most plausible candidate gene in the region for the observed effect at this locus is, however, the CXADR gene, located $98 \mathrm{~kb}$ downstream of the association signal. This gene encodes the Coxsackievirus and adenovirus receptor (CAR) protein, which is mostly expressed in the heart, brain and pancreas [15]. CAR has a long-recognised role as viral receptor in the pathogenesis of viral myocarditis and its sequela of dilated cardiomyopathy [16, 17]. Upon infection, the enteroviral (as is the Coxsackie $\mathrm{B}$ virus) protease $2 \mathrm{~A}$ cleaves dystrophin, resulting in disruption of the dystrophinglycoprotein complex, a component of the cytoskeleton of cardiac myocytes [18]. Interestingly, frequency of active Coxsackie B virus infection has been reported to be high in a group of MI patients who died suddenly [19]. The physiological role of CAR was initially less clear. It is expressed at the intercalated disc between cardiomyocytes, suggesting a role in conduction of the cardiac electrical impulse and cell-cell communication [20, 21]. Indeed, two studies have reported a physiological role for CAR in localisation of connexin 45 at the intercalated disks of atrioventricular node cardiomyocytes as well as its role in conduction of the cardiac impulse within this cardiac compartment [22, 23]. In addition, protein levels of connexin 43, which is expressed in atrial and ventricular myocytes and in distal parts of the conduction system, were reduced in CARdeficient mice, suggesting that loss of CAR could impact on ventricular conduction [23]. Thus, $C X A D R$ is a candidate gene for the association reported here.

However, providing support for a role of CXADR (or any other gene) in mediating the observed effect is a daunting task. The SNPs mediating the association signal on chromosome $21 \mathrm{q} 21$ are not linked to amino acid changing variants within the CAR coding region (or any other gene in the 
region). This precludes an effect through functional variation of a gene product, implying that the role of these SNPs in modulation of arrhythmia risk is to be sought in effects on gene expression. Effects of common genetic variation on gene expression is considered to be an important mechanism impacting on inter-individual variation in disease susceptibility, and many GWAS studies have in fact identified many signals within non-coding (intronic and intergenic) regions [24]. This will require studying gene expression as a function of genotype at this locus in the human heart.

\section{Conclusion and Future Directions}

It is clear that other genetic variation in other areas of the genome will impact on risk of VF during acute ischaemia. These loci, as for the one we identified, are expected to carry small effect sizes, implying that increased numbers of AGNES cases and controls will be required to uncover additional genes by GWAS. For this reason, recruitment of AGNES cases and controls is ongoing.

The risk carried by the common genetic variation we identified at chromosome $21 \mathrm{q} 21$ is small, meaning that genotyping of this locus alone for risk stratification in patients will not reach the accuracy that is considered as clinically informative. Nevertheless, if additional such loci are identified, one could envisage that by virtue of their additive effect, genotyping of such variants at multiple loci may in the future lead to their use in clinical practice. Importantly, however, the GWAS approach we used here has revealed a previously unsuspected involvement of chromosome 21q21 in arrhythmia risk. Further research on the mechanism of this locus will ultimately provide novel insight into arrhythmia mechanisms in this condition.

\begin{abstract}
Acknowledgements The authors are supported by research grants from the Netherlands Heart Foundation (2007B010 and 2007B202), the Interuniversity Cardiology Institute of the Netherlands (ICIN, 06402), the Center for Translational Molecular Medicine of the Netherlands (CTMM-COHFAR) and the Leducq Foundation (05CVD). Connie R. Bezzina is an Established Investigator of the Netherlands Heart Foundation (2005T024).
\end{abstract}

Open Access This article is distributed under the terms of the Creative Commons Attribution Noncommercial License which permits any noncommercial use, distribution, and reproduction in any medium, provided the original author(s) and source are credited.

\section{References}

1. Huikuri HV, Castellanos A, Myerburg RJ. Sudden death due to cardiac arrhythmias. N Engl J Med. 2001;345(20):1473-82.

2. Zipes DP, Wellens HJ. Sudden cardiac death. Circulation. 1998;98 (21):2334-51
3. Wilde AA, Bezzina CR. Genetics of cardiac arrhythmias. Heart. 2005;91(10):1352-8.

4. Watkins H, Ashrafian H, McKenna WJ. The genetics of hypertrophic cardiomyopathy: Teare redux. Heart. 2008;94 (10):1264-8.

5. Myerburg RJ. Sudden cardiac death: exploring the limits of our knowledge. J Cardiovasc Electrophysiol. 2001;12 (3):369-81.

6. Noseworthy PA, Newton-Cheh C. Genetic determinants of sudden cardiac death. Circulation. 2008;118(18):1854-63.

7. Friedlander Y, Siscovick DS, Weinmann S, et al. Family history as a risk factor for primary cardiac arrest. Circulation. 1998;97 (2):155-60.

8. Jouven X, Desnos M, Guerot C, et al. Predicting sudden death in the population: the Paris Prospective Study I. Circulation. 1999;99 (15):1978-83.

9. Dekker LR, Bezzina CR, Henriques JP, et al. Familial sudden death is an important risk factor for primary ventricular fibrillation: a case-control study in acute myocardial infarction patients. Circulation. 2006;114(11):1140-5.

10. Bodmer W, Bonilla C. Common and rare variants in multifactorial susceptibility to common diseases. Nat Genet. 2008;40(6):695-701.

11. Bezzina CR, Pazoki R, Bardai A, et al. Genome-wide association study identifies a susceptibility locus at 21q21 for ventricular fibrillation in acute myocardial infarction. Nat Genet. 2010;42 (8):688-91.

12. Agema WR, Monraats PS, Zwinderman AH, et al. Current PTCA practice and clinical outcomes in the Netherlands: the real world in the pre-drug-eluting stent era. Eur Heart J. 2004;25(13):116370.

13. Yoshida Y, Matsuda S, Ikematsu N, et al. ANA, a novel member of Tob/BTG1 family, is expressed in the ventricular zone of the developing central nervous system. Oncogene. 1998;16(20):268793.

14. Godbout R, Andison R, Katyal S, et al. Isolation of a novel cDNA enriched in the undifferentiated chick retina and lens. Dev Dyn. 2003;227(3):409-15.

15. Tomko RP, Xu R, Philipson L. HCAR and MCAR: the human and mouse cellular receptors for subgroup $C$ adenoviruses and group B coxsackieviruses. Proc Natl Acad Sci USA. 1997;94 (7):3352-6.

16. Bowles NE, Richardson PJ, Olsen EG, et al. Detection of CoxsackieB-virus-specific RNA sequences in myocardial biopsy samples from patients with myocarditis and dilated cardiomyopathy. Lancet. 1986;1(8490):1120-3.

17. Pauschinger M, Bowles NE, Fuentes-Garcia FJ, et al. Detection of adenoviral genome in the myocardium of adult patients with idiopathic left ventricular dysfunction. Circulation. 1999;99 (10):1348-54.

18. Badorff C, Lee GH, Lamphear BJ, et al. Enteroviral protease 2A cleaves dystrophin: evidence of cytoskeletal disruption in an acquired cardiomyopathy. Nat Med. 1999;5(3):320-6.

19. Andreoletti L, Venteo L, Douche-Aourik F, et al. Active Coxsackieviral B infection is associated with disruption of dystrophin in endomyocardial tissue of patients who died suddenly of acute myocardial infarction. J Am Coll Cardiol. 2007;50(23):2207-14.

20. Noutsias $M$, Fechner $H$, de Jonge $H$, et al. Human coxsackie-adenovirus receptor is colocalized with integrins alpha(v)beta(3) and alpha(v)beta(5) on the cardiomyocyte sarcolemma and upregulated in dilated cardiomyopathy: implications for cardiotropic viral infections. Circulation. 2001;104(3):275-80.

21. Shaw CA, Holland PC, Sinnreich M, et al. Isoform-specific expression of the Coxsackie and adenovirus receptor (CAR) in 
neuromuscular junction and cardiac intercalated discs. BMC Cell Biol. 2004;5(1):42.

22. Lim BK, Xiong D, Dorner A, et al. Coxsackievirus and adenovirus receptor (CAR) mediates atrioventricular-node function and connexin 45 localization in the murine heart. $\mathrm{J}$ Clin Invest. 2008;118(8):2758-70.
23. Lisewski U, Shi Y, Wrackmeyer U, et al. The tight junction protein CAR regulates cardiac conduction and cell-cell communication. J Exp Med. 2008;205(10):2369-79.

24. Cookson W, Liang L, Abecasis G, et al. Mapping complex disease traits with global gene expression. Nat Rev Genet. 2009;10 (3):184-94. 